


\title{
Conflictos mineros en el Perú: entre la protesta y la negociación
}

\author{
Maritza Paredes*
}

* Profesora asociada del Departamento de Ciencias Sociales, Sección Sociología, Pontificia Universidad Católica del Perú (PUCP). Correo electrónico: maritza.paredes@pucp.pe

Fecha de recepción: 01/07/19. Fecha de aceptación: 29/08/19 


\title{
Conflictos mineros en el Perú: entre la protesta y la negociación
}

\section{RESUMEN}

Este artículo realiza una revisión analítica de la literatura sobre los procesos de negociación que emergen de conflictos asociados a la minería, en particular en el Perú. Busca identificar los lineamientos teóricos cruciales que ofrece esta literatura. Estos lineamientos, ayudan a problematizar futuras investigaciones sobre los desafíos de estos procesos de negociación y su capacidad para superar el conflicto. Finalmente, a partir de estos lineamientos, el artículo presenta y discute brevemente los artículos del volumen Industrias extractivas, conflictos, negociaciones y diálogos en el Perú.

Palabras clave: conflicto, industrias extractivas, minería, negociación, diálogo.

\section{Mining conflicts in Peru: between protest and negotiation}

\begin{abstract}
This article carries on an analytical review of literature on negotiation and mining conflicts, particularly in Peru. It also seeks to identify important theoretical recommendations offered by this literature. These recommendations serve to problematize future research on the challenges of these negotiation processes and their ability to overcome disagreements. Finally, based on these recommendations, the article presents and discusses the articles of the volume Extractive Industries, conflicts, negotiations and dialogues in Peru.
\end{abstract}

Keywords: conflict, extractive industries, mining, negotiation, dialogue. 
¡No es Dios, papacitos: es la Cerro de Pasco Corporation! Manuel Scorza, Redoble por Rancas

\section{INTRODUCCIÓN}

La relación de la minería con las comunidades locales de su entorno es longeva y nunca ha estado libre de conflicto. Manuel Scorza narra una de las más excepcionales historias de esta relación conflictiva a mediados del siglo pasado en la sierra central del Perú. El autor cuenta que en Yanahuanca llega un día un ingeniero de la corporación minera, "un gringo". Poco tiempo después, los campesinos se dan cuenta de la llegada de más hombres y la construcción del «alambrado», que avanza, encerrando lagunas, cerros, pastizales y hasta los propios pueblos. Los pobladores ya no pueden alimentar a sus animales libremente y el camino de un pueblo a otro se vuelve incluso más largo, porque hay que rodear el «alambrado» de más de 100 kilómetros. Un viajero, que ha visto alambrados similares en otras partes, les anuncia que el alambrado no acaba. "Quieren cercar el mundo", les dice. Luego, los animales ya no solo se mueren por no tener pastos que comer, sino que se enferman por la contaminación y los pobladores también sufren cambios de color de piel. Ante la situación, los pobladores se organizan y luchan con los hombres de la mina y la guardia civil hasta la muerte.

El último ciclo del boom de precios de minerales (2004-2014) ha revivido una nueva versión del conflicto descrito por Scorza. Sin embargo, las oportunidades de diálogo y negociación surgen en este nuevo ciclo de una manera diferente. A diferencia de ciclos anteriores, ahora los conflictos mineros ocurren en un contexto global donde las demandas de los pobladores se inscriben en un entorno político —al menos formalmente- más favorable al diálogo. A pesar de que la violencia sigue estando presente, la extracción basada en la represión indiscriminada de los pobladores, como sucedió en Rancas, no parece posible, al menos no sin altos costos políticos para gobiernos y corporaciones.

En las últimas décadas, se ha establecido —sobre todo en el ámbito internacional - un conjunto de principios y declaraciones sobre el diálogo y la participación. Estas han sido promovidas mayormente por organismos internacionales y ONG, que subrayan la relevancia de que las inversiones privadas incorporen la participación y el diálogo de todos los stakeholders (empresa, Estado y sociedad civil) en la toma de decisiones (Lane y Corbett, 2005; O’Faircheallaigh, 2010)ํ․

\footnotetext{
1 En 1992, en la Conferencia de las Naciones Unidas sobre el Medio Ambiente y Desarrollo (Cnumad) se acordó enfatizar la importancia de la participación de las personas en la gestión ambiental. Fue la primera vez que se considera en este campo ambiental que el conocimiento y los
} 
Sin embargo, la forma como los gobiernos, empresas y comunidades han adoptado herramientas y políticas para hacer posibles estos procesos de negociación, y su impacto sobre los territorios en términos sociales o ambientales, han empezado a ser examinados y cuestionados (Dupuy, 2014; Hilson, 2012). Los estudios sobre estos procesos en el caso peruano y latinoamericano empiezan a producir un conjunto de «trazados» analíticos, que como en los artículos que se presentan en este volumen, permiten un mejor entendimiento de estos procesos de negociación y diálogo en el futuro.

El presente artículo tiene por objetivo realizar una revisión analítica de la literatura sobre los procesos de negociación que emergen de estos conflictos relacionados con la minería, en particular en el Perú. La finalidad es identificar los principales lineamientos teóricos que ofrece esta literatura sobre negociación y diálogo de conflictos vinculados con la minería. Además, a partir de estos lineamientos, en la última sección se presentan y discuten brevemente los artículos del presente volumen. Asimismo, esta introducción ofrece una sistematización que pretende incentivar la investigación analítica sobre los desafíos de estos procesos de negociación y su capacidad de superar el conflicto.

Los procesos de diálogo y negociación forman parte de procesos de conflictividad más amplios. Estos conflictos constituyen más que la ruptura de las relaciones entre las partes en tensión o el resquebrajamiento de las instituciones: en estos procesos se expresan pugnas de cómo gobernar la convivencia o la «co-producción» del territorio (Bebbington, 2008a). De allí radica la importancia de entender mejor lo que está en juego en estos procesos de acción colectiva y social relacionados con la actividad minera, bajo qué lógicas, en qué condiciones y con qué recursos actúan y toman decisiones los actores participan en procesos de negociación que emergen de los conflictos mineros.

Este artículo está organizado en cuatro secciones. La sección 2 presenta de forma general y breve la situación de conflictividad socioambiental en el Perú y los procesos de negociación que emergen en el contexto del conflicto minero en el Perú. La sección 3 aborda analíticamente la situación de oscilación o intermitencia entre protesta y negociación en la literatura del conflicto social. La sección 4 desarrolla una propuesta de cinco lineamientos para el estudio de los procesos de negociación.

valores de la población local debe tomarse en cuenta. Lo siguió el Pacto del Ecuador, promovido por la Naciones Unidas (1999). En cuanto a la minería, la formación en 1999 del Global Mining Iniciative (GMI) durante el Annual Meeting of the World Economic Forum celebrado en Suiza fue un hito en relación con el paradigma participativo. Esta iniciativa buscó sentar las bases para que la actividad minera genere un desarrollo sostenible y se creó la iniciativa «Mining, Minerals, and Sustainable Development (MMSD)». 
Finalmente, la sección 5 presenta los artículos de este volumen y su aporte crítico a esta problemática.

\section{La conflictividad socioambiental en el Perú}

En el Perú, la alta conflictividad ha sido registrada y sistematizada por la Defensoría del Pueblo (DP) desde el año 2004. Los datos actualizados de la DP muestran que los conflictos socioambientales constituyen el gran grueso de los conflictos $(68 \%)^{2}$, y dentro de este tipo, el subtipo relacionado con la minería es de $62 \%$ (ver gráfico 1).

El origen de estos conflictos ha sido ampliamente estudiado en el Perú ${ }^{3}$ y en otras partes de Latinoamérica (por ejemplo, Bebbington, Bebbington, Bury, Lingan, Muñoz y Scurrah et al.; Bebbington, ed., 2012; Delamaza, Maillet y Martínez Neira, 2017;, Dietz y Engels, 2017; Kirsh, 2007; Martínez-Allier, 2002; Svampa y Antonelli, 2009, entre otros). En un plano general, la literatura coincide en una acelerada expansión de la frontera de extracción de metales e hidrocarburos (Bebbington et al., 2008a; Larsen, 2018; Tsing, 2009). Este avance fue posible gracias a los altos precios internacionales, las favorables reformas institucionales de los Estados (Arellano-Yanguas, 2011) y los avances tecnológicos que hicieron posible la explotación y el transporte de minerales e hidrocarburos, antes no viables comercialmente (Bebbington et al., 2008a; Bury, 2005). Por ejemplo, la minería en Perú ha experimentado un crecimiento extraordinario desde la década de 1990. La expansión de la superficie asignada a la exploración minera aumentó de 4 millones a aproximadamente 24 millones de hectáreas entre 1993 y 1997 (De Echave, 2005, p. 10). Esta expansión de la industria minera en el Perú ha penetrado ecologías cada vez más frágiles y poblaciones más vulnerables (Orihuela, Huaroto y Paredes, 2014; Zegarra, Orihuela y Paredes, 2007).

Los conflictos socioambientales expresan fundamentalmente la emergencia de nuevos actores y disputas por la gestión de recursos, cada vez más escasos. Asimismo, estos ponen de manifiesto diferentes concepciones sobre el futuro, posibilidades de desarrollo y sostenibilidad (Bebbington, Hinojosa, Bebbington, Burneo y Warnaars, 2008b). Así, estas disputas en contextos mineros han dado lugar a espacios de diálogo y negociación, que han sido registrados por la DP (DP, 2019). De los conflictos activos, el $82 \%$ de los conflictos socioambientales se encuentra

\footnotetext{
2 Esta categoría se refiere al control, uso y/o acceso al ambiente y sus recursos. Están presentes también componentes políticos, económicos, sociales y culturales (DP, 2019). Otras definiciones que han recibido estos conflictos en la literatura son conflictos ecoterritoriales (Swampa, 2012) o ecológicos (Martínez Allier, 2003).

3 Entre las principales investigaciones destacan Arce (2015), Arellano-Yanguas (2011), De Echave, Diez, Revesz, Huber, Tanaka y Lanata (2009), Merino (2015), entre otros.
} 
siguiendo algún proceso de diálogo y negociación. Además, estos procesos tienden a iniciarse después de algún alto de violencia, que apunta a llamar la atención de las autoridades para que las demandas de las poblaciones sean escuchadas.

Gráfico 1. Conflictos en el Perú (ambientales, minería e hidrocarburos) 2007-2017

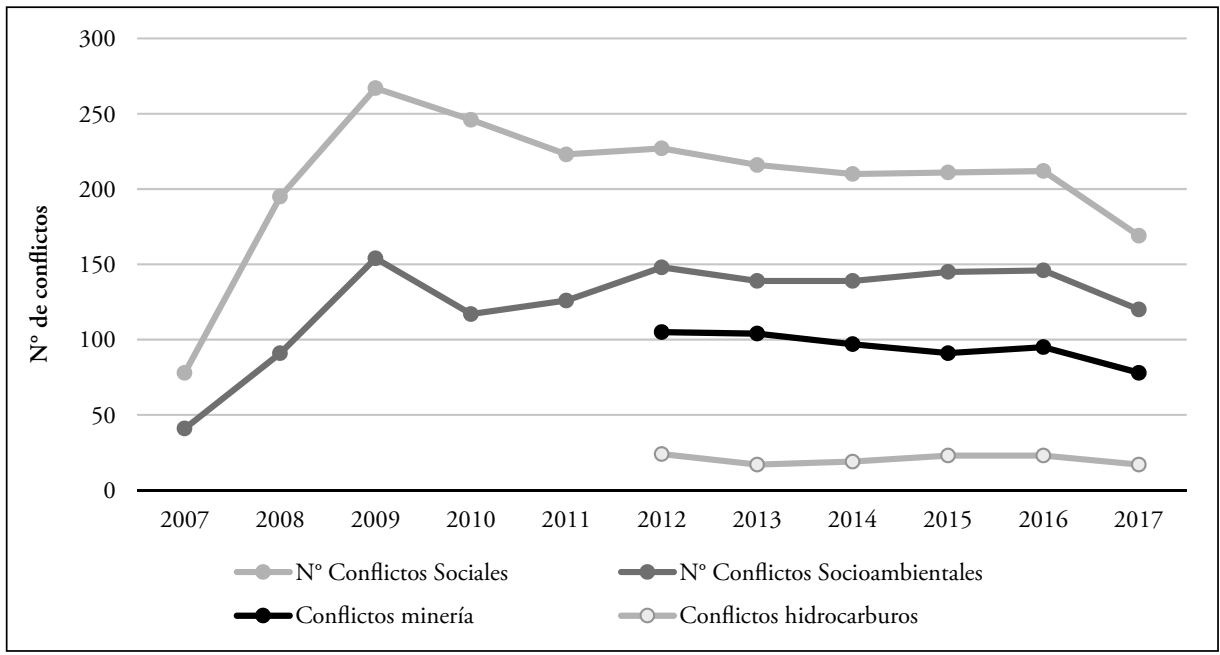

Fuente: Elaboración propia con base en los informes anuales Defensoría del Pueblo (2007-2017).

En este contexto, las "mesas de diálogo" se han institucionalizado como un mecanismo semiformal para canalizar la negociación. Según el informe de la Presidencia del Consejo de Ministros (PCM, 2013), estos espacios pueden establecerse (o no) a través de mecanismos legales (resoluciones ministeriales). Sin embargo, en la práctica no existe normatividad que regule estos procesos. Así, no hay criterios para la formación de estas mesas, las dinámicas de negociación, tiempos, toma de decisiones o acuerdos, o la selección de sus miembros (PCM, 2013). Las características de cada proceso de diálogo dependen de cada conflicto y de los actores involucrados, con resultados muy variados. En abril de 2019, la DP reportó que el $92 \%$ de los procesos de negociación se dan a través de estas mesas.

Adicionalmente, el crecimiento de esos conflictos y la demandas por diálogo llevó a la creación de funciones estatales para monitorear y gestionar el conflicto (Paredes y De la Puente, 2017). Por un lado, la DP ha sostenido su rol de monitoreo y prevención de conflicto y su intervención en un porcentaje alto de estos procesos de negociación: 84\% (DP, 2019). Por otro lado, la Oficina Nacional de Diálogo y Sostenibilidad (ONDS) de la Presidencia del Consejo de Ministros (PCM) fue creada en 2013 con la finalidad de atender los conflictos desde la PCM, con un enfoque de prevención, diálogo y solución pacífica de conflictos. 
Este alto número de casos activos en negociación denota un lado menos analizado del conflicto socioambiental: los procesos de negociación y diálogo que se inician a raíz de la protesta, e incluso de actos de violencia. Las comunidades impactadas por la minería han buscado negociar y «coproducir» las condiciones en que

Gráfico 2. Número de conflictos sociales activos en proceso de diálogo en el Perú (2008-2017)

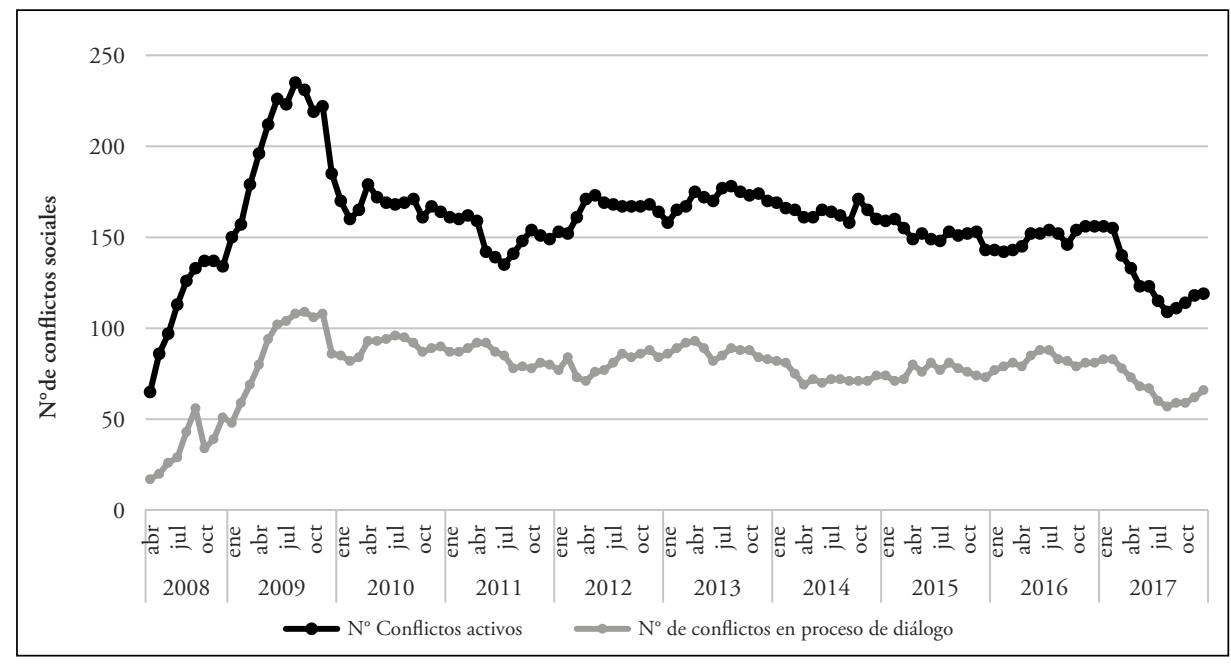

Fuente. Elaboración propia con base en informes anuales de la Defensoría del Pueblo (2008-2017).

se realizan las inversiones extractivas en sus territorios a través de la movilización y la protesta (Bebbington, 2007a). En algunos casos, incluso, se ha sostenido que los procesos de negociación disminuyen la tensión y logran establecer algunos acuerdos que permiten la convivencia de los actores locales con las empresas mineras (De Echave et al., 2009).

\section{EL CONFLICTO SOCIAL TRANSFORMATIVO}

El conflicto social expresa «la lucha por valores o demandas de estatus, poder y recursos escasos» (Coser, 1956, p. 8) ${ }^{4}$ en una amplia gama de ámbitos de la vida social 5 . Los conflictos mineros representan una expresión de las fuertes asimetrías

\footnotetext{
4 Traducción propia.

5 Estas disputas no se limitan a la economía y la posición de clase - como fue inicialmente planteado por Marx - sino que pueden ser étnico-raciales, religiosos, comunitarios, de género, territoriales, entre otros.
} 
de poder y decisión sobre el uso de los recursos naturales, sus beneficios y consecuencias, tan características de América Latina y, particularmente de las economías basadas en recursos naturales. Asimismo, el análisis del conflicto social plantea una mirada crítica a un enfoque de armonía social, donde no se hacen visibles o se encubren las tensiones subyacentes de poder en las relaciones sociales, así como la estratificación social que estas tensiones producen. Esta realidad de competencia entre grupos sociales por la distribución de recursos escasos y del poder y la búsqueda de soluciones se presenta en la sociedad de una forma más frecuente que anómala, señala Collins (1975), por lo cual el conflicto social «tranformativo» constituye una aproximación "realista» y no una opción normativa necesariamente.

La reacción de los actores a esta tensión y su acción social; sin embargo, constituye un fenómeno social diferente en el que concurren múltiples posibilidades (McAdam y Schaffer, 2012). La producción de agencia requiere que la situación de injusticia sea percibida y entendida como ilegitima por quienes vayan a emprender una acción (Collins, 1975), y una reflexión de costos y beneficios cuando esta es colectiva (Olson, 2009) ${ }^{6}$. En el siglo pasado, la menor conflictividad de la minería con las comunidades de su entorno pudo deberse a una mayor legitimidad de las relaciones entre compañías mineras y comunidades campesinas. La tecnología de socavón absorbía significativamente la mano de obra de la comunidad campesina y ofrecía a sus trabajadores en el campamento minero educación formal, centros de salud y un mercado local que estimulaba el área rural circundante. Así, el «pueblo minero» funcionaba como un símbolo y promesa de modernidad anhelada (Ballard y Banks, 2003, p. 292), a pesar de la contaminación, el riesgo laboral y la alta desigualdad en los campamentos mineros (Flores Galindo, 1983).

La nueva minería de tajo abierto ha reducido radicalmente el uso de mano obra no calificada. Los campamentos mineros se volvieron espacios cerrados y exclusivos para los pocos trabajadores externos muy calificados (Salas, 2008) y la minería, en general, perdió legitimidad ante la comunidad, revelándose más claramente las tensiones de poder subyacentes. La movilización, sin embargo, no es automática: depende de las condiciones internas, (organización, recursos, etc.) (McCarthy y Zald, 1977) o externas (oportunidad política) que tengan las comunidades (McAdam, Tarrow y Tilly 2001). Los programas de Responsabilidad Social Corporativa (RSC) y la «licencia social» para operar surgen, precisamente, como una respuesta de la industria minera a esta oposición local y un mecanismo de recuperar la legitimidad del sector (Dashwood, 2012; De Sa, 2018; Hilson, 2012).

\footnotetext{
6 También las emociones, la angustia o la rabia, pueden tener un rol importante, en ciertas etapas de la acción colectiva contenciosa (Jasper, 2011).
} 
Por otro lado, la población podría haber percibido también que el poder de la minería era «incontestable». Una «reflexión de costos y beneficios» que, siguiendo a Moore (1978, p. 500), puede producir una sensación «inevitabilidad» y de «expropiación de la indignación moral» (Moore, 1978) ${ }^{7}$. Esto lleva a la desafección colectiva. Sin embargo, un nuevo contexto de reconocimiento de derechos, y su difusión entre las comunidades impactadas por la minería, debilitarían esa sensacion de «inevitabilidad», aumentando la disposición por la movilización. Por ejemplo, la expansión de los derechos humanos e indígenas, el conocimiento de estos derechos por las comunidades (transferida por actores como ONG y el propio Estado), la información sobre otros conflictos similares en todo el mundo y sus repertorios (referendos populares, apelación a derecho internacional, entre otros), y la presencia de coaliciones transnacionales de incidencia y apoyo (conocimiento, recursos, contactos etc.).

El conflicto social en las circunstancias planteadas arriba puede tomar múltiples formas de acción colectiva y repertorios. El estudio de la política contenciosa, muy importante en los últimos cincuenta años (Tilly y Tarrow, 2015; Mc Adam, Tarrow y Tilly 2001; Tarrow, 1994), ha sido influyente en el análisis de los conflictos mineros, cuya literatura ha puesto la atención en los repertorios de movilización y en la forma como los actores en estos conflictos encuentran oportunidades políticas para movilizarse y producir nuevas identidades colectivas y políticas ${ }^{8}$. Menos énfasis se ha puesto en estudiar cómo se manejan estas tensiones cuando no existe una clara o abierta protesta, o cuando la protesta es seguida por desmovilización (Auyero, 2009), o por un proceso de negociación y búsqueda de diálogo y acuerdos (Anguelovski, 2011; De Echave et al., 2009; Bebbington et al., 2008a; 2008b) ${ }^{9}$.

A partir de la aún creciente literatura empírica sobre la negociación, en el Perú y en el ámbito internacional, este artículo recoge el aspecto "transformador» del conflicto. El análisis enmarca el conflicto minero en una teoría transformativa y de cambio social. Es decir, se busca entender no solo la acción política contenciosa y transgresora (McAdam, Tarrow and Tilly 2001) que emerge del conflicto, sino aquella acción colectiva que busca negociación y diálogo simultáneamente. En este marco conceptual, el conflicto social denota más que la ruptura de las relaciones entre las partes en tensión o el resquebrajamiento de las instituciones, sino sus cualidades transformativas. Según Coser, el conflicto social denota fundamentalmente un carácter "productivo", es un motor del cambio social (1956, p. 198).

\footnotetext{
Agradezco esta idea al revisor(a) anónimo (a).

8 Por ejemplo, parten de este marco analítico Arce (2015), De Echave et al. (2009), Haarstad y Fløysand (2007), Jaskoski (2014), McDonell (2015), Bebbington (2007), Paredes (2006, 2007), entre otros.

9 McAdam y Schaffer (2012) plantean que esta es una limitación general a la literatura de los movimientos sociales.
} 
Coser cuestionó el significado del conflicto social como una «enfermedad», tal como era concebido por el funcionalismo parsoniano ${ }^{10}$. Más bien, siguiendo a Simmel (1903, p. 490), Coser planteó que el conflicto social era un mecanismo fundamental de la sociedad de resolución de tensiones y de cambio social. Para otros autores, el conflicto social es incluso lo opuesto a la violencia política y no busca la destrucción del otro, sino más bien lograr nuevos arreglos (Wieviorka, 2009).

Los conflictos relacionados con la minería, al revelar tensiones existentes entre diferentes posiciones e intereses sociales entre compañías mineras y comunidades, al mismo tiempo pueden constituir oportunidades de innovación o transformación institucional (Bebbington, 2012). La literatura más reciente sobre el conflicto en general plantea que la negociación y el diálogo, vistos desde una perspectiva trasformativa, son mecanismos que promueven el entendimiento y el logro de acuerdos consensuados entre las partes en disputa (Lederach, 2014). Sin embargo, también hay un balance menos optimista que se enfoca en las diferentes motivaciones de los actores involucrados, y pone en cuestión que se estén resolviendo los conflictos relacionados con las dimensiones intangibles de las dinámicas sociales, como la cultura y valores respecto de la naturaleza y los recursos; o se esté realmente incluyendo en la toma de decisiones la voz, conocimiento local, y visiones de desarrollo de grupos tradicionalmente marginados, como mujeres, pueblos indígenas y campesinos (Kapoor, 2001; O'Faircheallaigh, 2010).

\section{EL ESTUdio DE LOS PROCESOS SOCIALES DEL DIÁLOGO Y NEGOCIACIÓN}

La examinación empírica de los procesos de diálogo y negociación en el contexto del conflicto minero permitirá entender mejor cómo estos procesos pueden ser transformativos del conflicto o no. Para ello, se requiere una problematización conceptual sobre los aspectos críticos subyacentes al diálogo y la negociación, que necesitan ser revelados y examinados en los casos de investigación. Esto, en particular, porque estos conflictos en los contextos mineros se dan entre actores con diferentes recursos y posiciones de poder y en territorios con limitadas capacidades estatales. En esta sección se analizan cinco trazos conceptuales que aparecen en la literatura como subyacentes a los procesos de diálogo y negociación en estos contextos. Estos trazos conceptuales se refieren a (i) la legitimidad de los procesos; (ii) las asimetrías de poder en las interacciones; (iii) las múltiples racionalidades de los actores; (iv) la hibridez de los procesos y acuerdos, y (v) la capacidad del Estado y el legado institucional.

10 Coser cuestionó directamente la postura defendida por Parsons, que concebía al conflicto como generador de consecuencias principalmente disruptivas, disociativas y destructivas de la vida social (1956, p. 21). 


\section{La legitimidad del proceso de diálogo}

La literatura sobre procesos de diálogo y negociación en el Perú resalta la importancia de la voluntad de las partes como crucial para construir vínculos de confianza entre los actores que participan en los procesos de negociación (Espluga, Prades, Gamero y Solà, 2009; Ormachea, Caravedo, Moreno y Bedoya, 2014). En su estudio sobre el relativo éxito de la negociación en el Perú en el contexto minero, Crabtree, Gómez, Hurtado, Paredes, Reyes-Hurt, Thorp y Zambrano (2019) señalan que las actitudes fueron claves para el éxito del diálogo. Resaltan en su análisis que las minas Goldfields (en Cajamarca) y San Rafael (en Puno) mantuvieron mejores procesos de diálogo, porque mantuvieron la voluntad de diálogo desde el inicio del proyecto y ello se volvió una práctica permanente.

Sin embargo, considerando una larga tradición de imposición y abuso en las actividades mineras, el diálogo puede deslegitimarse rápidamente. Los procesos de negociación y diálogo no necesariamente son instrumentos neutrales y, por lo tanto, no siempre conducen a un mejor gobierno de los conflictos (Fontana y Grugel, 2016). Estos procesos de diálogo muchas veces son invocados o aceptados como actos "performativos» para mostrar que existe la intención de dialogar y dar solución a los problemas. Sin embargo, el diálogo solo es utilizado para legitimar los proyectos extractivos ya decididos (Schilling-Vacaflor y Flemmer, 2015). En una entrevista de una investigación en curso, una de las informantes de comunidad de Alto Huancané señaló que "el diálogo sirve solo para apaciguar el conflicto»" ${ }^{11}$.

La literatura ha resaltado, por ejemplo, la frecuente baja disposición (o capacidad) de las autoridades y las empresas de cumplir los acuerdos como producto del diálogo. Por un lado, no existen reglas de juego claras para hacer seguimiento a la implementación de los acuerdos, y por otro lado, existen indicios de que en muchas ocasiones no hay una voluntad real de los actores estatales y de la empresa de implementar los acuerdos (Huamaní, Macassi, Alegría y Rojas, 2012, p. 27). Este tipo de acciones plantean la necesidad de entender que el diálogo ocurre como un proceso "performativo» en lugar de "transformativo». En estos casos, los actores (funcionarios del Estado y las compañías) gestionan «expresiones», actos y palabras, pero sin ofrecer efectivas salidas a los problemas de las comunidades o una verdadera participación en la toma de decisiones ${ }^{12}$.

En suma, el diálogo "performativo» es problemático porque deslegitima el diálogo y la negociación, lo que le quita su potencial transformador. Como señaló

\footnotetext{
11 Entrevista en Alto Huacané, diciembre, 2015, parte de la investigación «Participación tóxica» dirigida por Maritza Paredes, Ciencias Sociales, PUCP.

12 Basándose en el concepto de «manejo de impresiones» de Goffman (1959), Lapegna (2016) define a este tipo de ejercicio político como "gobernanza performativa».
} 
la informante de Espinar, si el diálogo solo es buscado para «apaciguar» el conflicto social y desmovilizar a los actores que reclaman, probablemente se mitigará el conflicto en el corto plazo, pero ni lo resuelve ni lo transforma, y volverá a ser fuente de problemas.

\section{Las asimetrías de poder en las interacciones}

La existencia de asimetrías de poder e información entre los actores empresariales, del Estado y las comunidades del entorno minero ha sido planteada como problemática para el diálogo y la negociación transformativa. Mucha de la literatura más aplicada y específica al diseño de contextos de diálogo ha señalado la necesidad de elaboración de adecuadas estrategias de diálogo y un buen diseño de los espacios de negociación. Esto para superar la desconfianza entre actores, pérdida de legitimidad de líderes, asimetrías estructurales y la debilidad organizacional, entre otros factores (Crabtree et al., 2019; Ormachea et al., 2014, pp. 363-370). El dilema reside en que, si bien los procesos de negociación y diálogo son planteados como la «solución al problema», el problema que subyace es la dificultad de desarrollar un diálogo «deliberativo» desde diferentes posiciones de poder (Habermas, 1990). En lo que sigue, se distingue la discusión de la literatura sobre las asimetrías de poder con respecto al diálogo ambiental, de la literatura sobre asimetrías en otros aspectos del diálogo. Esto por la abundancia y pertinencia de la primera en la generación de conflictos sociales.

\section{Diálogo e impactos ambientales inciertos}

La situación de asimetría entre actores en este tipo de espacios de diálogo se presenta también cuando los espacios de discusión y negociación se nutren de marcos normativos que privilegian (o imponen) el lenguaje técnico científico (Li, 2015). En el caso de conflictos socioambientales complejos, como los mineros, gran parte de lo que se negocia son efectos inciertos y un conjunto de incertidumbres sobre el futuro de las comunidades ${ }^{13}$. Las experiencias previas de proyectos extractivos han generado percepciones negativas sobre los impactos de la minería en las poblaciones y, por lo tanto, cuestionan la certeza del conocimiento técnico y científico para mitigar y controlar esos impactos. Esta desconfianza en la ciencia para mitigar el «riesgo» es una característica de la sociedad industrial contemporánea, en la cual la producción de riqueza está correspondida por una producción similar — si no mayor- de riesgos (Beck, 1998).

13 Por ejemplo, para el proyecto Conga se propuso la reubicación de las lagunas Perol, Cortada o Mala, Azul y Chica, las cuales serían trasvasadas a los reservorios Chailhuagón, Perol, Inferior y Superior que construiría Minera Yanacocha. 
Los actores demandantes son condicionados en espacios de diálogo donde se busca trasladar los cuestionamientos técnicos al ámbito público. Sin embargo, se intenta restringir el diálogo al ámbito netamente técnico, con lo cual este queda eliminado de la influencia del debate político (Callon, Lascoumes y Barthe, 2009; Li, 2015). Varios conflictos mineros expresan los cuestionamientos de las poblaciones potencialmente afectadas a este tipo de diálogo, donde se encuentran en una posición de desigualdad (ver Gil, 2009; Paredes, 2008; Yrivarren; 2017) Callon et al. (2009) plantean que esta asimetría es característica de nuevos «espacios híbridos de diálogo» ${ }^{14}$ (por su variedad de participantes), donde los ciudadanos regulares cuestionan no solo la legitimidad técnica de los expertos, sino la legitimidad moral de este tipo de conocimiento, que se les quiere imponer como concluyentes. Esto lo hacen a partir de la confrontación entre conocimientos y experiencias fracasadas de la aplicación de los conocimientos técnicos y científicos en contraste de conocimientos, intereses y experiencias locales (Callon et al., 2009, p. 27).

Esto hace que los conflictos socioambientales constituyan un escenario de tensión recurrente, donde la protesta y diálogo transitan entre incertidumbres sociales y técnicas que se perpetúan en el tiempo, en espacios generalmente dominados por actores que exigen una discusión en un lenguaje técnico (Li, 2015, p. 22). Por el contrario, el reconocimiento de la dimensión social del tema técnico restaura la posibilidad de un diálogo también político, tomando en cuenta a todos los actores y reconociendo la naturaleza incierta de los efectos (Callon et al., 2009, p. 27).

Entonces, el conflicto social plantea una serie de condiciones para ser transformativo, sobre todo cuando este diálogo implica información compleja. La literatura señala algunas condiciones, como (i) la comprensión por todos los actores de la información que se transmite; (ii) el diálogo crítico sobre esta información sin imposiciones sobre su legitimidad técnica o científica — considerando que existen muchas posibilidades y efectos inciertos-; (iii) la opinión de otros expertos, y (iv) la formación de los actores de su propia opinión del tema (De la Puente, 2017; Paredes y De La Puente, 2015; Schilling-Vacaflor y Flemmer, 2015). Adicionalmente, el tipo de lenguaje utilizado es importante, dado que cuando es técnico y legalista impide que los grupos consultados puedan participar de manera activa

14 Callon et al. (2009) definen un foro híbrido como «espacios abiertos donde los grupos pueden reunirse para discutir opciones técnicas relacionadas con el colectivo, [siendo] híbrido porque los grupos involucrados y los portavoces que afirman representarlos son heterogéneos, incluidos expertos, políticos, técnicos, y laicos que se consideran involucrados. También son híbridos porque las preguntas y los problemas que se tratan se abordan en diferentes niveles en una variedad de dominios, desde la ética hasta la económica, incluida la fisiología, la física nuclear y el electromagnetismo» (p. 18) (traducción propia). 
y dificulta la construcción de confianza y consenso duradero (De la Puente, 2017; Schilling-Vacaflor y Flemmer, 2015) ${ }^{15}$.

\section{Asimetrías en otros aspectos del diálogo}

A menudo, la priorización de los temas que deben tomarse en cuenta responde a la agenda de los actores más poderosos, y se excluyen de esta elección a los que menos poder tienen. Schilling y Flemmer (2015) muestran cómo, en el caso de la consulta previa (CP) en el Perú y Bolivia, el proceso de consulta no incluyó los temas que eran importante para los participantes, por ejemplo, la titulación de sus tierras o el acceso de las comunidades a agua potable. Muchas veces, el diálogo es restringido a los posibles impactos futuros, buscando aislar «los proyectos de inversión» del conjunto integral de problemas, muchos previos y estructurales, que las comunidades ya enfrentan y que pueden tener incidencia en esos impactos.

Otra forma de expresión de las asimetrías se refiere a cómo y quién decide el momento donde se consulta, dialoga o se negocian los proyectos de inversión, que a menudo son susceptibles de modificaciones en el tiempo. Esto significa que la población y las organizaciones civiles solo participan en la evaluación de una etapa del proyecto, o alguna medida de aquel. Sin embargo, la población es excluida de las otras etapas de programación o gestión de los proyectos (Kapoor, 2001). Los diálogos y negociaciones en minería se dan en el marco de la CP y la discusión del Estudio de Impacto Ambiental (EIA). En ambos, la participación está diseñada para un momento del ciclo del proyecto. Sin embargo, las inversiones están sujetas a cambios y situaciones inesperadas por las fluctuaciones de precios que cambian la capacidad financiera de los proyectos, la propiedad y las medidas de mitigación. La principal razón por la que se evita la participación a lo largo del proyecto es porque las compañías y el Estado consideran que la gestión participativa implica un gran compromiso de tiempo y recursos (humanos, institucionales y financieros) para garantizar participación de las partes interesadas (Kapoor, 2001).

La asimetría puede darse también dentro de las mismas comunidades afectadas que buscan negociar. Existe dentro de las comunidades procesos de «micropoder» que se ocultan a los actores fuera de la comunidad. Salas (2008) ha descrito estos problemas y sus impactos en la negociación de las comunidades con las empresas mineras. Así, algunos participantes pueden ser más influyentes que otros, por diferentes razones, y manipular las deliberaciones participativas para sus propios fines. Estos participantes más poderosos pueden también tergiversar las posiciones,

\footnotetext{
15 Si las decisiones se concentran en debates netamente técnicos en torno a grupo de expertos, se construyen acuerdos que no son sostenibles ante la desconfianza por la comunidad (De la Puente, 2017; Huamaní et al., 2012).
} 
emplear evidencia falsa o uso retórico del lenguaje para persuadir, influir o silenciar al resto de participantes (Holmes y Scoones, 2000; Tewdwr-Jones y Allemendinger, 1998). Algunos autores señalan la necesidad de hacer visibles estas desigualdades, pero que además estas desigualdades estén abiertas al cuestionamiento, el diálogo y la negociación entre las partes interesadas (Nelson y Wright, 1995). El reconocimiento de las desigualdades de poder los participantes puede inducirlos a idear controles y balances, por ejemplo, al instituir una representación más alta de lo normal para los grupos socioeconómicos desfavorecidos (Kapoor, 2001, p. 274).

Los «mediadores» $\mathrm{o}$ «asesores» que cumplen «actores terceros», tales como ONG, instituciones públicas u observadores internacionales, pueden aportar soporte técnico y estrategias de relacionamiento que ayudan a la reducción de asimetrías en el diálogo (Irigoyen y Chávez, 2017; Portocarrero, Sanborn y Camacho, 2007; Ríos-Delgado, 2016). Sin embargo, también se requiere cambiar la naturaleza más sustancial del diálogo desde su diseño hasta la culminación del proyecto. Para hacer significativa la negociación y el diálogo, se requiere nada menos que un cambio en la cultura organizacional del Estado y las empresas. Esto involucra pensar los procesos de explotación extractiva como procesos que hay que evaluar en un sentido más amplio que el estrictamente empresarial, con procesos más flexibles y con objetivos a largo plazo, procedimientos, resultados y horizontes de más amplio alcance. Estos cambios de comportamiento, a su vez, dependen mucho de cambios estructurales y políticos que implican liderazgo y voluntad política y el establecimiento de marcos legales apropiados, entre otros. A menudo, estos cambios no se realizan si no están significativamente apoyados por cambios administrativos y consensos políticos (Kapoor, 2001).

\section{Múltiples racionalidades de los actores}

Gran parte de la producción sobre negociación y diálogo en el contexto minero revela la importancia de enfocarse en la dimensión cultural para analizar los conflictos y darles solución. Esto implica entender las lógicas de negociación de los actores y cómo estas lógicas se expresan en las relaciones entre ellos. Asimismo, Huamaní (2015) señala que es importante hacer explícito lo importante dentro las cosmovisiones de los actores, cómo estos expresan la frustración y sus necesidades insatisfechas.

En general, la literatura ha ido revelando que el problema de la interculturalidad es un problema muy complejo que no se reduce al uso de los idiomas indígenas en los procesos de negociación. Si bien el tema del lenguaje es indispensable, el problema es de carácter ontológico y los supuestos ontológicos que subyacen los conflictos y las negociaciones. Este tema ha tomado impulso en los estudios medioambientales con el cuestionamiento de que en todas las culturas existe un paradigma de dualidad 
entre «sociedad y naturaleza» (Descola, 2011; Latour, 2007). En este sentido, Blaser (2009), De la Cadena (2010), y Salas (2019) han señalado que lo que está en juego en conflictos relacionados con el medio ambiente y la naturaleza no significa necesariamente lo mismo para diferentes horizontes culturales. Las publicaciones de Fabiana Li (2017) y Guillermo Salas (2019) son probablemente los estudios más destacados sobre el Perú desde este enfoque.

Por un lado, Li (2017) muestra cómo diferentes lógicas y valores de «equivalencia» coexisten entre comunidades indígenas, campesinos, analistas, los burócratas estatales, sociedad civil, entre otros. Estas lógicas se desarrollan respecto de las propiedades de los cuerpos naturales que van a ser negociados y coexisten de forma subyacente en los procesos de conflicto y diálogo minero. En el caso de los estudios ambientales de la mina Conga de Yanacocha en Cajamarca, los técnicos y expertos de la mina crearon «equivalencias» entre el agua de un manantial que alimenta un canal de riego y el agua extraída por la mina para uso agrícola, que se libera en el mismo canal después de ser tratada químicamente. Asimismo, indicaron qué conocimiento e información cuenta o era válido para hacer esta equivalencia, por su carácter técnico u «objetivo». Li muestra que, para los campesinos, los técnicos y expertos de Yanacocha hicieron conmensurable dos visiones del agua y del Estado diferentes e impusieron una lógica de equivalencia que fue cuestionada por ellos. Los comuneros manifestaban que usaban el agua del canal para la agricultura, pero también para otros usos; además, esta agua de manantiales era clave para el funcionamiento de otros cuerpos hídricos y el funcionamiento del ecosistema natural integrado. Así, Li concluye que este tipo de desencuentros resultan en la proliferación de disputas en lugar de acuerdos.

Por otro lado, Salas (2019), plantea que, en los horizontes culturales de las comunidades andinas indígenas, los apus son las montañas reales, seres materiales vivos y no espíritus inmateriales que habitan en ellos. La agencia de estas montańas no es un tema de creencia o religión, sino una manera de entender cómo funciona o es el mundo. Las montańas activas no forman parte de un mundo «sobrenatural», tampoco son parte de mundo «natural» exclusivamente. Las personas interactúan con estos «seres de la tierra» en los contextos más mundanos y cotidianos ${ }^{16}$. La obtención de alimentos, por ejemplo, de la tierra implica negociaciones con estos «seres». Salas señala que, en diferentes niveles, cualquier tipo de ofrenda de alimentos realizada por el pueblo andino aborda este tema de negociación y pagos a los seres de la tierra. La extracción subterránea de metales implica la mutilación de las montańas y poner en riesgo su fertilidad; la negociación entre el minero y la montańa es requerida para que esta no se resista a ser minada y para aminorar

16 De la Cadena (2010) se refiere a este concepto como earthbeings. 
los riesgos dentro de ella. En un claro contraste con las operaciones subterráneas, donde la negociación es crucial para minar las montańas, la minería a cielo abierto literalmente elimina montañas enteras y las destruye. La minería a cielo abierto consiste en procesar el cadáver de los seres de la tierra, dice Salas (2019), extraer el metal y dejar el resto como escombros contaminados.

Las implicancias de estas lógicas para las discusiones y controversias en el contexto minero son importantísimas. En particular porque plantean defensas locales de articulación entre las poblaciones y la naturaleza que son muy localizadas (De la Cadena 2010) y que superan el mero carácter instrumental sobre los recursos naturales (Escobar 1995). Lamentablemente, estas lógicas a menudo resultan invisibles para las personas no indígenas, incluido el personal de la mina y del Estado. A la luz de estas investigaciones, el diálogo intercultural tan invocado en estas negociaciones adquiere un carácter más complejo que el de exclusivamente tener traductores o poner la información en los idiomas de las comunidades

\section{La hibridación de los procesos}

Callon et al. (2009) plantean que los «fórums híbridos» —espacios abiertos de discusión técnica de actores heterogéneos- pueden ser espacios profundamente asimétricos. Otro elemento de estos foros de diálogo híbridos es que ponen en tela de juicio la hegemonía del Estado como la fuente más importante de toma de decisiones en el campo del uso del territorio, el uso de recursos naturales, y en general, el gobierno de las industrias extractivas. Esto porque las comunidades afectadas se movilizan y se oponen a los proyectos, obligando tanto al Estado como a las empresas a negociar. La participación de nuevos actores (Estado, sociedad civil, ONG, instancias internacionales) que desempeñan un papel decisivo en la toma de decisiones, que incluso pueden llegar a reemplazar al Estado, nos lleva a la discusión de la negociación como un proceso híbrido. Este nuevo tipo de sistema híbrido da lugar al uso de instituciones privadas (mercados) e incentivos (económicos, sociales) como nuevas formas de gobernanza de los conflictos extractivos y ambientales (Armitage, de Loë y Plummer, 2012).

Estos sistemas híbridos ponen en cuestión las capacidades del Estado para enfrentar problemas como los conflictos socioambientales y revelan la mayor presión de los ciudadanos por una mayor participación en la toma de decisiones y los beneficios esperados (a pesar de los costos de transacción) en la solución de estos. Los costos de transacción aumentan debido a que los procesos deliberativos involucran más perspectivas y diferentes tipos de conocimiento (De Loë y Kreutzwiser, 2007). Este cambio ha generado un conjunto de oportunidades para que emerjan modelos participativos de gobernanza híbridos, involucrando a actores estatales y no estatales que cooperan para lograr objetivos compartidos ganen legitimidad. 
Sin embargo, hay que tener en cuenta que este cambio también refleja un impulso por la descarga de la responsabilidad estatal en la gobernanza de los recursos (Bäckstrand, Kahn, Kronsell, Lövbrand y Elgar, 2010). Además, como se ha señalado en las subsecciones anteriores, estos mecanismos de participación híbridos no generan necesariamente mejores resultados en la solución de los conflictos. En torno al conflicto extractivo, los mecanismos de negociación se dividen en dos. Por un lado, se encuentran aquellas que se dan dentro de espacios y con procedimientos reglamentados por la ley, en la que el Estado peruano y la sociedad deliberan sobre asuntos públicos (DP, 2019). Por otro lado, existen los espacios creados por la voluntad de las partes, en los que una diversidad de actores confluye y negocia sobre diversos temas y agendas, siendo principalmente estos las mesas de diálogo (DP, 2019). Los espacios creados por los actores - los cuales no necesariamente se encuentran oficializados - pueden ser considerados como instituciones informales, como en el caso de las «mesas de diálogo»"

Si bien, las mesas de diálogo cumplen una función complementaria a la estatal y no compiten con mecanismos creados por el Estado, el problema con la ausencia de regulación es que pueden generar situaciones en que los mismos actores estatales optan por no responder a los acuerdos a pesar de tener la capacidad para hacerlo (Holland, 2016). Como señalábamos, en la primera dimensión conceptual, en estas ocasiones algunos actores pueden optar por gestos «performativos» en los procesos de diálogo, pero sin resultados efectivamente transformativos, redundando en la situación de desigualdad y conflicto social (Furze, De Lancy y Birckhead, 1996; Richards, 1995).

\section{La capacidad del Estado y el legado institucional}

De manera transversal, la literatura reconoce que el Estado y los gobiernos locales, a pesar de ser actores políticos centrales durante los procesos de conflicto, se caracterizan por una serie de carencias institucionales en su participación en los procesos de negociación, priorizando el restablecimiento del orden, la protección de la propiedad y la iniciativa privada, y reforzando percepciones de colusión con las empresas mineras (Zeisser, 2015). Además, resalta la continuidad de un sistema de gestión de conflictos débil, centralizado, desarticulado, únicamente activo en situaciones de crisis (Huamaní, 2010; Huamaní y Macassi, 2011; Tanaka, 2012; Vela Clavo, 2014). Sobre este punto, se llama la atención sobre la ausencia de normativa clara en materia de conflictos sociales, así como políticas estatales de gestión del conflicto

17 Helmke y Levitsky (2004) señalan que las instituciones informales son aquellas «reglas» socialmente compartidas, usualmente no escritas, que son creadas, comunicadas, e impuestas fuera de los canales oficialmente sancionados (pp. 11-12). 
contradictorias. Estas conclusiones refuerzan la importancia del involucramiento del Estado - principalmente del gobierno central— en los procesos de negociación, no solo como mediador de los intereses entre comunidad y empresa minera, sino como un potencial garante para el cumplimiento y viabilidad técnica de los acuerdos que resulten de estos procesos (Balbuena y Ramos, 2017; Bedoya, 2013).

Si bien diversos estudios muestran el fracaso de los procesos de negociación por incumplimiento de acuerdos, desconfianza de actores locales, falta de liderazgo e incapacidad del Estado y normativa poco desarrollada (Flores, 2016), la dimensión política, principalmente de los gobiernos locales, puede encaminar trayectorias alternativas a un escalamiento del conflicto si cuentan con la legitimidad suficiente. Por ejemplo, Hurtado (2016) y el Banco Mundial (2015) señalan que es posible establecer acuerdos y alcanzar una experiencia positiva si se atiende el conflicto en su estado latente y con el liderazgo de los gobiernos regionales para la promoción de espacios de diálogo. No obstante, esta situación suele ser muy poco frecuente: se presentan casos donde incluso se excluye al Estado de estos procesos de negociación, entendiéndose como un espacio de "particulares» (Camacho y Lossio, 2007) ${ }^{18}$. En suma, es necesario examinar mejor la interacción entre conflictos socioambientales y construcción estatal. Es importante no solo examinar las diferentes formas de organización de la sociedad civil, sino también las diferentes estructuras organizativas de los organismos estatales que van construyéndose, la permeabilidad de las organizaciones estatales y las condiciones en las cuales surgen (o no) compromisos duraderos entre Estado y sociedad.

La literatura también aborda la mayor complejidad de estos procesos de negociación, dado que se enmarcan en contextos locales con cierto legado histórico que pueden ser influyentes al momento de entender el conflicto; es decir, estas dinámicas se reflejan finalmente en los procesos de negociación. Por ejemplo, un grupo de estudios pone énfasis en las trayectorias económicas de las comunidades y su relación con otros actores locales (empresa minera y gobiernos locales) como elementos clave al momento de entender intereses contrapuestos en las mesas de diálogo, principalmente vinculadas con los procesos de decisión sobre el uso de recursos (Alcántara, 2018), mientras que otro grupo de estudios explora la tensión entre la negociación y la protesta como una dinámica acumulativa para el establecimiento de acuerdos a partir de continuas demandas sociales y ambientales por la sociedad civil (Arotoma, 2013). Asimismo, aunque de manera indirecta, estudios sobre las relaciones de convivencia y gobernanza entre empresa minera y comunidad muestran algunas vistas sobre los efectos y desafíos que los procesos

18 En Tintaya, ni los dirigentes de las organizaciones de base ni la empresa minera quisieron al débil Estado en la mesa de negociaciones (De Echave, 2005). Similares conclusiones extrae Salas (2008) a partir de estudiar el proceso de compras de tierras de puna por Antamina. 
de negociación generan sobre las organizaciones comunales (Burneo de la Rocha y Ortiz de Zevallos, 2010).

\section{CONTENIDO DEL VOLUMEN Y REFLEXIONES FINALES}

Los artículos de este volumen presentan de manera muy comprensiva varios de los aspectos conceptuales mencionados en la sección anterior del artículo y nos ayudan a ilustrar como estos aspectos pueden promover o limitar el diálogo y la negociación y la transformación del conflicto.

Lorena de la Puente, en su artículo "No estuvimos a la altura de la situación": límites de la participación ciudadana en la mesa de diálogo de Espinar (2012-2013)», plantea los problemas de la transformación del conflicto si el diálogo pierde legitimidad al tener características performativas y llevarse adelante sin atender las asimetrías de poder entre los actores. En su análisis de la más reciente mesa de diálogo de Espinar, el resultado final fue que el diálogo no fue percibido como satisfactorio por todos los actores involucrados, sobre todo por los más afectados, las comunidades campesinas. Se describe cómo la propia organización y metodología de la mesa de diálogo en Espinar reprodujo condiciones asimétricas entre los participantes. Por un lado, los menores recursos humanos (dirigentes) de las comunidades sobrecargó el trabajo en unos pocos dirigentes de las comunidades campesinas que hacían el trabajo voluntariamente, mientras que los miembros del Estado y la empresa recibían una remuneración por su participación. Por otro lado, el lenguaje técnico relacionado con los problemas medioambientales alienó a la población campesina cuando esta era la principal afectada. Pese al apoyo de ONG y la Iglesia local, las decisiones fueron tomadas sin mayor comprensión de la población de las comunidades, lo que generó confrontaciones posteriores con respecto a la aprobación del Informe Final

Mauricio Zavaleta, en su artículo "¿¿Dieciséis céntimos a cambio de aguas ácidas?” Respuestas políticas a la expansión minera en Cajamarca», examina los casos de protesta en Combayo (2006) y Conga (2011-2012) en Cajamarca, buscando discutir la racionalidad detrás de la población que se organiza políticamente y resiste la expansión de las fronteras de la extracción minera en la región. El autor señala que, hoy, la menor capacidad de oferta laboral de la mina para la población campesina centra la negociación y el diálogo alrededor de los beneficios directos locales otorgados por la empresa minera como compensación de la actividad y el uso e impacto sobre los recursos naturales. Predomina una lógica de evaluación de los costos, del deterioro de los recursos naturales, que, a su vez, se perciben mayores que los beneficios obtenidos por los programas sociales y servicios de la mina. Para el autor, la racionalidad de los campesinos que se resisten a la expansión de la minería es pragmática y efectiva, donde la población campesina cuantifica la 
afectación de sus medios de subsistencia con el fin de demandar una compensación equivalente. El artículo plantea y revalúa la noción de equivalencias al momento de las negociaciones. En estas equivalencias entran también las incertidumbres respecto de los costos ambientales y aquellos que se pueden generar por la actuación del Estado. En este contexto, el rechazo a la expansión de la frontera minera se acrecienta y la organización de coaliciones en contra se inicia.

Aarón Quiñón, en su artículo «¿El pasado importa? Legados institucionales, sociedad civil y burocracia local para la gestión de conflictos socioambientales en Moquegua (2000-2016)», examina casos de relativo éxito en la negociación y diálogo. En su análisis del caso de Moquegua, el autor va a sugerir que los antecedentes institucionales desarrollados, previos al contexto del boom de recursos y el legado de participación ciudadana, fueron claves para el relativo éxito del diálogo con la minería en Moquegua. La gestión del conflicto fue positiva en tanto en las tres localidades existía una relación previa entre la burocracia local y sociedad civil, además de experiencia y legados institucionales que promovían una acción social reconocida para canalizar las demandas. El liderazgo y organizaciones fuertes, con experiencia y repertorios institucionales en Moquegua, además de respaldo en organizaciones locales, previamente constituidos, constituyeron las bases de un diálogo con potencialidad de llegar a consensos.

Finalmente, Laura Álvarez, en su artículo "Asambleando el mundo. La experiencia de la Unión de Asambleas de Comunidades en las luchas socioambientales en Argentina», plantea una argumentación diferente desde el contexto argentino. $\mathrm{Su}$ artículo explica el surgimiento de la Unión de Asambleas Comunitarias en Argentina, red que reúne a distintas organizaciones y asambleas que abordan problemáticas socioambientales y que tuvo como origen un movimiento ciudadano en contra del inicio de la etapa de explotación de un proyecto minero en la Patagonia argentina. A diferecia de los casos peruanos, este artículo plantea cómo, a raíz de un proceso de participación y diálogo que culminó en un referéndum en el que participaron los pobladores locales para definir el inicio del proyecto Esquel, se crearon condiciones transformativas de cómo tratar «la cuestión minera» como una problemática social relevante en la agenda pública más nacional. A diferencia de los casos peruanos, este último artículo plantea de manera exploratoria las características transformativas de los conflictos mineros con relación a la construcción de nuevos recursos sociales y no solo la gestión de los casos mismos.

En suma, estos cinco artículos del volumen plantean una reflexión sobre el conflicto desde preguntas que giran alrededor de la potencialidad transformadora del conflicto, fundamentalmente vía la generación de espacios de diálogo y negociacion. Sin embargo, esta potencialidad está llena de desafios complejos que no anulan las oportunidades que abre el diálogo, pero que tampoco garantizan sus resultados. 
Estos desafios no son solo procedimentales, aunque los procedimientos son importantes. Los desafíos implican formas de relacionamiento político complejo, que incluyen desde poner en cuestion la voluntad real y ontología dominante con las que se dialoga, hasta la actual legitimidad de acuerdos históricos, como la autoridad nacional de los recursos naturales, que hoy día se ven subvertidos por comunidades locales que defienden sus territorios.

\section{REFERENCIAS BIBLIOGRÁFICAS}

Alcántara Ruiz, C. R. (2018). Trayectorias económicas y su influencia en la dinámica del conflicto social en la sierra de La Libertad. Lima: Pontificia Universidad Católica del Perú.

Almaric, F. (1999). Natural resources, governance and social justice. World Development, 43, 732-745.

Anguelovski, I. (2011). Understanding the dynamics of community engagement of corporations in communities: The Iterative relationship between dialogue processes and local protest at the Tintaya Copper Mine in Peru. Society and Natural Resources, 24(4), 384-399. https://doi.org/10.1080/08941920903339699

Arce, M. (2015). La extracción de recursos naturales y la protesta social en el Perú. Lima: Fondo Editorial de la Pontifica Universidad Católica del Perú.

Arellano-Yanguas, J. (2011). ¿Minería sin Fronteras? Conflicto y desarrollo en regiones mineras del Perú. Lima: OXFAM, IEP.

Armitage, D., De Loë, R. y Plummer, R. (2012). Environmental governance and its implications for conservation practice. Conservation Letters, 5(4), 245-255. https://doi. org/10.1111/j.1755-263X.2012.00238.x

Arotoma Rojas, I. (2013). La negociación por el Proyecto Minero Quellaveco en la Región Moquegua (2000-2012). Lima: Pontificia Universidad Católica del Perú.

Bäckstrand, K., Kahn, J., Kronsell, A., Lövbrand, E. y Elgar, E. (2010). Environmental Politics and Deliberative Democracy. Examining the Promise of New Modes of Governance. https://doi.org/10.4337/9781849806411

Balbuena, P. y Ramos, G. (2017). Gestión del cumplimiento de los acuerdos derivados de los conflictos sociales: un modelo de análisis desde la gestión pública y las políticas públicas. Politai: Revista de Ciencia Politica, 8(15), 81-92.

Ballard, C. y Banks, G. (2003). Resource Wars: The Anthropology of Mining. Annual Review of Anthropology, 32(1), 287-313. https://doi.org/10.1146/annurev.anthro. 32.061002 .093116

Banco Mundial (2015). Construyendo desde el conflicto: Las mesas de diálogo de Tintaya y Moquegua en Perú. Washington D.C.

Bebbington, A. (2007a). Elementos para una ecología política de los movimientos sociales y el desarrollo territorial en zonas mineras. En A. Bebbington (ed.), Minería, movimientos sociales y respuestas campesinas: una ecología politica de transformaciones territoriales (pp. 23-46). Lima: Instituto de Estudios Peruanos/Centro Peruano de Estudios Sociales. 
Bebbington, A. (ed.). (2007b). Minería, movimientos sociales y respuestas campesinas: una ecologia politica de transformaciones territoriales. Lima: IEP-CEPES.

Bebbington, A. (ed.). (2012). Social Conflict, Economic Development and Extractive Industry: Evidence from South America. New York: Taylor \& Francis Group. https://doi. org/10.4324/9780203639030

Bebbington, A. y Bebbington, D. (2009). Actores y ambientalismos: conflictos socioambientales en Perú. Íconos, 35(septiembre), 117-128. https://doi.org/10.17141/ iconos.35.2009.371

Bebbington, A., Bebbington, D., Bury, J., Lingan, J., Muñoz, J. P. y Scurrah, M. (2008). Mining and Social Movements: Struggles Over Livelihood and Rural Territorial Development in the Andes. World Development, 36(12), 2888-2905. https://doi. org/10.1016/j.worlddev.2007.11.016

Bebbington, A., Hinojosa, L., Bebbington, D., Burneo, M. L. y Warnaars, X. (2008). Contention and Ambiguity: Mining and the Possibilities ofDevelopment. Development and Change, 39(6), 887-914. https://doi.org/10.1111/j.1467-7660.2008.00517.x

Bedoya, C. (2013). La nueva propuesta del Estado para el abordaje de conflictos sociales: La Oficina Nacional de Diálogo y Sostenibilidad. Revista Argumentos, 7(1), 10-13. Recuperado de http://revistaargumentos.iep.org.pe/wp-content/uploads/2014/04/ bedoya_marzo2013.pdf

Blackburn, J. y Holland, J. (eds.). (1998). Who Changes? Institutionalizing Participation in Development. Londres: Intermediate Technology Publications. https://doi.org/ $10.3362 / 9781780446417$

Blaser, M. (2009). The threat of the Yrmo: The political ontology of a sustainable hunting program. American Anthropologist, 111(1), 10-20. https://doi.org/10.1111/j.15481433.2009.01073.x

Buchanan, K. S. (2013). Contested discourses, knowledge, and socio-environmental conflict in Ecuador. Environmental Science \& Policy, 30, 19-25. https://doi.org/10.1016/j. envsci.2012.12.012

Burneo de la Rocha, M. L. y Ortiz de Zevallos, A. C. (2010). Poder, comunidades campesinas e industria minera: el gobierno comunal y el acceso a los recursos en el caso de Michiquillay. Anthropologica, 28(1), 85-110. https://doi.org/10.1016/089570619(50)0405X-

Bury, J. (2005). Mining mountains: Neoliberalism, land tenure, livelihoods, and the new Peruvian mining industry in Cajamarca. Environment and Planning A, 37(2), 221-239. https://doi.org/10.1068/a371

Callon, M., Lascoumes, P. y Barthe, Y. (2009). Acting in an Uncertain World. An essay on technical democracy. London: MIT Press.

Camacho, L. y Lossio, F. (2007). La Mesa de Diálogo de la provincia de Espinar, Cusco: Solucionando conflictos entre la sociedad civil y la empresa minera BHP Billiton Tintaya sin la presencia del Estado. En A. Panfichi (ed.), Participación ciudadana en el Perú: disputas, confluencias y tensiones (pp. 147-229). Lima: Pontificia Universidad Católica del Perú. 
Collins, R. (1975). Conflict Sociology: Toward an Explanatory Science. Recuperado de https:// www.jstor.org/stable/3339762? origin=crossref

Coser, L. (1956). The Functions of Social Conflict. Nueva York: The Free Press.

Cousins, B. (1997). How Do Rights Become Real? Formal and Informal Institutions in South Africa's Land Reform. IDS Bulletin, 28(4), 59-68. https://doi.org/10.1111/ j.1759-5436.1997.mp28004007.x

Crabtree, J., Gómez, T., Hurtado, E., Paredes, M., Reyes-Hurt, A., Thorp, R. y Zambrano, G. (2019). Diálogo y derechos humanos en el sector minero. Algunas lecciones del Perú. Lima.

Dashwood, H. (2012). The Rise of Global Corporate Social Responsibility. Nueva York: Cambridge University Press. https://doi.org/10.1017/CBO9781139058933

De Echave, J. (2005). Hacia una estimación de los efectos de la actividad minera en los indices de pobreza en el Perú. Lima: CooperAcción.

De Echave, J., Diez, A., Revesz, B., Huber, L., Tanaka, M. y Lanata, R. (2009). Minería y conflicto social. Lima: Cipca, IEP, CIES, Centro de Estudios Regionales Andinos Bartolomé de Las Casas.

De La Cadena, M. (2010). Indigenous cosmopolitics in the Andes: Conceptual reflections beyond "politics». Cultural Anthropology, 25(2), 334-370. https://doi.org/ 10.1111/j.1548-1360.2010.01061.x

De la Puente, L. (2017). Cuando el diálogo es parte del problema. La institucionalización de las mesas de diálogo en el Perú: El caso de conflicto de Espinar. Lima: Departamento de Ciencias Sociales. Pontificia Universidad Católica del Perú.

de Loë, R. C. y Kreutzwiser, R. (2006). Challenging the Status Quo: The evolution of Water Governance in Canada. En K. Bakker (ed.), Eau Canada. The Future of Canada's Water (pp. 85-103). Vancouver: University of British Columbia Press.

De Sa, P. (2018). Mining and sustainable development: territorializing the mining industry. Mineral Economics, 32, 131-143. https://doi.org/10.1007/s13563-018-0149-8

Defensoría del Pueblo. (2004-2019). Informe mensual de conflictos sociales.

Delamaza, G., Maillet, A. y Martínez Neira, C. (2017). Socio-territorial conflicts in Chile: Configuration and politicization (2005-2014). European Review of Latin American and Caribbean Studies, 104(jul-dic), 23-46. https://doi.org/10.18352/erlacs.10173

Descola, P. (2011). Más allá de la naturaleza y de la cultura. En L. Montenegro (ed.), Cultura y naturaleza. Aproximaciones a propósito del bicentenario de la independencia de Colombia (pp. 75-98). Bogotá: Jardín Botánico de Bogotá.

Dietz, K. (2017). Politics of Scale and Struggles over Mining in Colombia. En B. Engels y K. Dietz (eds.), Contested Extractivism, Society and the State (pp. 127-148). https:// doi.org/10.1057/978-1-137-58811-1_6

Dupuy, K. E. (2014). Community development requirements in mining laws. Extractive Industries and Society, 1(2), 200-215. https://doi.org/10.1016/j.exis.2014.04.007

Engels, B. y Dietz, K. (eds.). (2017). Contested Extractivism, Society and the State. Struggles over Mining and Land. https://doi.org/10.1057/978-1-137-58811-1

Escobar, A. (1995). El desarrollo sostenible: diálogo de discursos. Ecología Política, 9, 7-25. 
Espluga, J., Prades, A., Gamero, N. y Solà, R. (2009). El papel de la «confianza» en los conflictos socioambientales. Política y Sociedad, 46(1-2), 255-273.

Falleti, T. G. y Riofrancos, T. N. (2018). Endogenous Participation: Strengthening Prior Consultation in Extractive Economies. World Politics, 70(1), 86-121. https://doi. org/10.1017/S004388711700020X

Flores Galindo, A. (1983). Los mineros de la Cerro de Pasco, 1900-1930. Lima: Fondo Editorial de la Pontificia Universidad Católica del Perú.

Fontana, L. B. y Grugel, J. (2016). The Politics of Indigenous Participation Through «Free Prior Informed Consent»: Reflections from the Bolivian Case. World Development, 77(April 2015), 249-261. https://doi.org/10.1016/j.worlddev.2015.08.023

Furze, B., De Lancy, T. y Birckhead, J. (1996). Culture, conservation, and biodiversity: the social dimension of linking local level development and conservation through protected areas. Michigan: John Wiley \& Sons.

Garcia-Zamor, J.-C. (ed.). (1985). Public Participation In Development Planning And Management: Cases From Africa and Asia. Westview Replica Edition.

Gil, V. (2009). Aterrizaje minero: cultura, conflicto, negociaciones y lecciones para el desarrollo desde la minería en Áncash, Perú. Recuperado de http://dialnet.unirioja.es/servlet/ articulo?codigo $=5042131$

Goffman, E. (1959). The Presentation of Self in Everyday Life. Nueva York: Doubleday.

Haarstad, H. y Fløysand, A. (2007). Globalization and the power of rescaled narratives: A case of opposition to mining in Tambogrande, Peru. Political Geography, 26(3), 289-308. https://doi.org/10.1016/j.polgeo.2006.10.014

Helmke, G. y Levitsky, S. (2004). Informal Institutions and Comparative Politics: A Research Agenda. Perspectives on Politics, 2(4), 725-740. https://doi.org/10.1017/ S1537592704040472

Hilson, G. (2012). Corporate Social Responsibility in the extractive industries: Experiences from developing countries. Resources Policy, 37(2), 131-137. https://doi.org/ 10.1016/j.resourpol.2012.01.002

Holmes, T. y Scoones, I. (2000). Participatory environmental policy processes: Experiences from North and South (N.o Working Paper N¹13).

Hooland, A. (2016). Forbearance. American Political Science Review, 110(2), 232-246. https://doi.org/10.1017/S0003055416000083

Huamaní, G. (2010). Diagnóstico y propuesta de mejora del sistema nacional y regional de gestión de conflictos socio-ambientales. Diagnóstico nacional. Lima.

Huamaní, G. (2015). Sobre las herramientas para el análisis de los conflictos sociales. En L. Puma y C. Bedoya (eds.), Transformación de conflictos. Aportes al análisis y abordaje de conflictos para el desarrollo sostenible (pp. 13-27). Lima: ProDiálogo, Universidad Antonio Ruiz de Montoya.

Huamaní, G. y Macassi, S. (2011). Gestión de conflictos socio ambientales. Lima.

Huamaní, G., Macassi, S., Alegría, J. y Rojas, T. (2012). Hacia una mejor gestión de los conflictos socioambientales en el Perú. Lima: Consorcio de Investigación Económica y Social. 
Hurtado Banchero, M. F. (2016). Analisis de la "Mesa de Diálogo entre la sociedad civil de Moquegua, el Estado y la empresa Anglo American Quellaveco 2011- 2012”. Lima: Pontificia Universidad Católica del Perú.

Irigoyen, M. del R. y Chávez, J. A. (2017). El Grupo de Diálogo, Minería y Desarrollo Sostenible (GDMDS) como experiencia democrática deliberativa en los procesos socioambientales del Perú, en el siglo XXI. Lima: Pontificia Universidad Católica del Perú.

Jaskoski, M. (2014). Environmental licensing and conflict in peru's mining sector: A pathdependent analysis. World Development, 64, 873-883. https://doi.org/10.1016/j. worlddev.2014.07.010

Jasper, J. M. (2011). Emotions and social movements: Twenty years of theory and research. Annual Review of Sociology, 37, 285-303. https://doi.org/10.1146/annurev-soc081309-150015

Kapoor, I. (2001). Towards participatory environmental management? Journal of Environmental Management, 63(3), 269-279. https://doi.org/10.1006/jema.2001.0478

Kirsch, S. (2007). Indigenous movements and the risks of counterglobalization: Tracking the campaign against Papua New Guinea's Ok Tedi mine. American Ethnologist, 34(2), 303-321. https://doi.org/10.1525/ae.2007.34.2.303

Lane, M. B. y Corbett, T. (2005). The tyranny of localism: Indigenous participation in community-based environmental management. Journal of Environmental Policy and Planning, 7(2), 141-159. https://doi.org/10.1080/15239080500338671

Lapegna, P. (2016). Soybeans and Power: Genetically Modified Crops, Environmental Politics, and Social Movements in Argentina. Oxford University Press. https://doi. org/10.1093/acprof:oso/9780190215132.001.0001

Latour, B. (2005). Reassembling the Social: An Introduction to Actor-Network-Theory. Oxford: Oxford University Press.

Lederach, J. P. (2014). The Little Book of Conflict Transformation. Nueva York: Good Books. Li, F. (2015). Unearthing Conflict: Corporate Mining, Activism, and Expertise in Peru. Durham: Duke University Press. https://doi.org/10.1215/9780822375869

Li, F. (2017). Desterrando el conflicto. Empresas mineras, activistas y expertos en el Perú. Lima: IEP.

Martinez-Alier, J. (2002). The environmentalism of the poor: a study of ecological conflicts and valuation. https://doi.org/10.5860/CHOICE.40-4110

McAdam, D. y Schaffer, H. (2012). Putting Social Movements in their Place: explaining opposition to energy projects in the United States, 2000-2005. Cambridge: Cambridge University Press. https://doi.org/10.1017/CBO9781139105811

McAdam, D., Tarrow, S. y Tilly, C. (2001). Dinamycs of Contention. Cambridge: Cambridge University Press. https://doi.org/10.1017/CBO9780511805431

McCarthy, J. D. y Zald, M. N. (1977). Resource mobilization and social movements: A partial theory. American Journal of Sociology, 82(6), 1212-1241. https://doi. org/10.1086/226464 
McDonell, E. (2015). The co-constitution of neoliberalism, extractive industries, and indigeneity: Anti-mining protests in Puno, Peru. Extractive Industries and Society, 2(1). https://doi.org/10.1016/j.exis.2014.10.002

Merino Acuña, R. (2015). The Extractive Industries and Society The politics of extractive governance: Indigenous peoples and socio-environmental conflicts. Extractive Industries and Society, 2(1), 85-92. https://doi.org/10.1016/j.exis.2014.11.007

Montgomery, J. (1988). Bureaucrats and People: Grassroots Participation in Third World Development. Washington D.C.: Johns Hopkins University Press.

Muńoz, I., Paredes, M. y Thorp, R. (2007). Group Inequalities and the Nature and Power of Collective Action: Case Studies from Peru. World Development, 35(11), 19291946. https://doi.org/10.1016/j.worlddev.2007.01.002

Nelson, N. y Whright, S. (1995). Power and Participatory Development. Theory and Practice. ITDG Publishing. https://doi.org/10.3362/9781780445649

O'Faircheallaigh, C. (2010). Public participation and environmental impact assessment: Purposes, implications, and lessons for public policy making. Environmental Impact Assessment Review, 30(1), 19-27. https://doi.org/10.1016/j.eiar.2009.05.001

Olson, M. (2009). The logic of collective action (Vol. 124). Harvard University Press.

Orihuela, J. C., Huaroto, C. y Paredes, M. (2014). Escapando de la maldición de los recursos local: conflictos socioambientales y salidas institucionales. Lima.

Ormachea Choque, I., Caravedo, J., Moreno, G. y Bedoya, C. (2014). Minería, conflicto social y diálogo. Lima: ProDiálogo.

Paredes, M. (2008). Weak Indigenous Politics in Peru (CRISE Working Paper N³3). Oxford.

Paredes, M. (2016). The glocalization of mining conflict: Cases from Peru. Extractive Industries and Society, 3(4), 1046-1057. https://doi.org/10.1016/j.exis.2016.08.007

Paredes, M. y De la Puente, L. (2014). Protestas y negociaciones socio-ambientales. El caso de las industrias extractivas. En G. Damonte \& G. Vila (eds.), Agenda de investigación en temas socioambientales en el Perú: una aproximación desde las ciencias sociales (pp. 75-105). Lima: Cisepa-PUCP.

Paredes, M. y De la Puente, L. (2017). The Social Construction of a Public Problem: The Role of the Ombudsman in Building Institutions for Extractive Conflict. En E. Dargent, J. C. Orihuela, M. Paredes y M. E. Ulfe (eds.), Resource Booms and Institutional Pathways. The Case of the Extractive Industry in Peru (pp. 119-151). New York: Palgrave Macmillan. https://doi.org/10.1007/978-3-319-53532-6_5

Portocarrero, F., Sanborn, C. y Camacho, L. A. (eds.). (2007). Moviendo montañas: empresas, comunidades y ONG en las industrias extractivas. Lima: Centro de Investigación de la Universidad del Pacífico.

Presidencia del Consejo de Ministros (2013). Mesas de Diálogo, Mesas de Desarrollo y Conflictos Sociales en el Perú (Reporte Willaqniki N05.).

Richards, P. (2001). Participatory rural appraisal: A Quick and Dirty Critique. PLA Notes, 24. Ríos Delgado, T. (2016). Estrategias de Negociación y Gestión de Conflictos como Herramienta para los Gerentes. Gestión en el Tercer Milenio, 19(38), 7-12. 
Salas Carreño, G. (2008). Dinámica social y minería: familias pastoras de puna y la presencia del proyecto Antamina (1997-2002). Lima: Instituto de Estudios Peruanos.

Salas Carreńo, G. (2019). Lugares parientes. Comida, cohabitación y mundos andinos. Lima: Fondo Editorial de la Pontificia Universidad Católica del Perú.

Schilling-Vacaflor, A. y Flemmer, R. (2015). Conflict Transformation through Prior Consultation? Lessons from Peru. Journal of Latin American Studies, 47(04), 811-839. https://doi.org/10.1017/S0022216X15000826

Scott, J. (1998). Seeing Like a State. How Certain Schemes to Improve the Human Condition Have Failed. New Haven y London: Yale University Press.

Shepherd, A. (1995). Participatory environmental management: Contradiction of process, project and bureaucracy in the Himalayan foothills. Public Administration and Development. https://doi.org/10.1002/pad.4230150503

Simmel, G. (1903). The Sociology of Conflict. American Journal of Sociology, 9(6), 798-811. https://doi.org/10.1086/211272

Svampa, M. (2012). Hacia una gramática de las luchas en América Latina: dimensiones de la acción y giro eco-territorial. Nostromo 5 (Otońo 2011-Primavera 2012): 77-93.

Svampa, M. y Antonelli, M. (2009). Minería transnacional, narrativas del desarrollo y resistencias sociales. Buenos Aires: Biblos.

Tanaka, M. (2012). ¿Por qué el Estado no responde adecuadamente a los conflictos sociales? ¿Qué hacer al respecto? Economía y Sociedad - CIES, 79 (marzo), 36-43.

Tarrow, S. (1994). Power in Movement Social Movements and Contentious Politics. Cambridge: Cambridge University Press.

Tewdwr-Jones, M. y Allmendinger, P. (1998). Deconstructing communicative rationality: a critique of Habermasian collaborative planning. Environment and Planning $A$, 30(11), 1975-1989. https://doi.org/10.1068/a301975

Tilly, C. y Tarrow, S. (2015). Contentious Politics. Oxford University Press, 53(9), 1689-1699. https://doi.org/10.1017/CBO9781107415324.004

Tsing, A. (2003). Natural Resources and Capitalist Frontiers. Economic and Political Weekly, 38(48), 5100-5106.

Vela Clavo, J. (2014). ¿Qué estamos entendiendo por "diálogo" ?: el estudio de la mesa de diálogo de Huari como una aproximación a la intervención estatal en los conflictos sociales. Lima: Pontificia Universidad Católica del Perú.

Wieviorka, M. (2009). Violence A Hew Approach. SAGE Publications.

Yrivarren, J. (2017). Ruido politico y silencio técnico: un ensayo sobre la discusión socioambiental de minas Conga. Lima: Punto Cardinal Editores.

Zazueta, A. (1995). Policy Hits the Ground: Participation and Equity in Environmental PolicyMaking. Washington D.C.: World Resources Institute.

Zegarra Méndez, E., Orihuela, J. C. y Paredes, M. (2007). Minería y economía de los hogares en la sierra peruana: impactos y espacios de conflicto (Documento de trabajo $\mathrm{N}^{\circ}$ 51). Lima: Grade.

Zeisser, M. y Al, E. (2015). La experiencia de la mesa de diálogo en Espinar 2012-2013: ¿Un nuevo modelo de gestión de conflicto socio ambiental? CooperAcción, 104. 